Mon. Not. R. Astron. Soc. 000, 000-000 (0000) Printed 1 July $2018 \quad$ (MN LATEX style file v2.2)

\title{
Formation, orbital and thermal evolution, and survival of planetary-mass clumps in the early phase of circumstellar disk evolution
}

\author{
Yusuke Tsukamoto ${ }^{1}$, Masahiro N. Machida ${ }^{2}$ and Shu-ichiro Inutsuka ${ }^{1}$ \\ ${ }^{1}$ Department of Physics, Nagoya University, Furo-cho, Chikusa-ku, Nagoya, Aichi, Japan \\ ${ }^{2}$ Department of Earth and Planetary Sciences, Kyushu University, 6-10-1 Hakozaki, Higashi-ku, Fukuoka, Fukuoka, Japan
}

1 July 2018

\begin{abstract}
We report the results of our three-dimensional radiation hydrodynamics simulation of collapsing unmagnetized molecular cloud cores. We investigate the formation and evolution of the circumstellar disk and the clumps formed by disk fragmentation. Our simulation shows that disk fragmentation occurs in the early phase of circumstellar disk evolution and many clumps form. The clump can be represented by a polytrope sphere of index $n \sim 3$ and $n \gtrsim 4$ at central temperature $T_{c} \lesssim 100 \mathrm{~K}$ and $T_{c} \gtrsim 100$ $\mathrm{K}$, respectively. We demonstrate, numerically and theoretically, that the maximum mass of the clump, beyond which it inevitably collapses, is $\sim 0.03 M_{\odot}$. The entropy of the clump increases during its evolution, implying that evolution is chiefly determined by mass accretion from the disk rather than by radiative cooling. Although most of the clumps rapidly migrate inward and finally fall onto the protostar, a few clumps remain in the disk. The central density and temperature of the surviving clump rapidly increase and the clump undergoes a second collapse within 1000 - 2000 years after its formation. In our simulation, three second cores of masses $0.2 M_{\odot}, 0.15 M_{\odot}$, and $0.06 M_{\odot}$ formed. These are protostars or brown dwarfs rather than protoplanets. For the clumps to survive as planetary-mass objects, the rapid mass accretion should be prevented by some mechanisms.
\end{abstract}

Key words: star formation - circum-stellar disk - methods: hydrodynamics smoothed particle hydrodynamics - protoplanetary disk - planet formation

\section{INTRODUCTION}

Stars form in molecular cloud cores. When the angular momentum in the core is non-negligible, circumstellar disk formation is inevitable because most of the gas cannot directly fall onto central protostar. According to theoretical studies on the gravitational collapse of unmagnetized molecular cloud cores, the protostar is surrounded by a circumstellar disk immediately after its formation (Bate 1998; Machida, Inutsuka \& Matsumoto 2010; Tsukamoto \& Machida 2011; Bate 2011).

As noted by Inutsuka, Machida \& Matsumoto (2010), the resulting disk should be sufficiently massive to develop gravitational instability (GI). If Toomre's $Q$ value of the disk is $\lesssim 1.5$, the disk is gravitationally unstable to non-axisymmetric perturbation and develops spiral arms Laughlin \& Bodenheimer 1994). These spiral arms readjust the surface density (Takahashi. Inutsuka \& Machida 2013) and raise the disk temperature, thereby re-stabilizing the disk. However, if radiative cooling is effective enough or the mass accretion onto the disk is sufficiently high, the disk may fragment and form clumps (Gammie 2001; Rice. Lodato \& Armitage 2005; $\quad$ Inutsuka, Machida \& Matsumoto 2010; Stamatellos, Whitworth \& Hubber 2011; Kimura \& Tsuribe 2012).

Disk fragmentation is a candidate mechanism of wideorbit planet formation (Rice, Lodato \& Armitage 2005; Vorobyov \& Basu 2010; Machida. Inutsuka \& Matsumoto 2011; Vorobyov, DeSouza \& Basu 2013). A wideorbit planet is a planet separated from the central star by more than 10 AU (Marois et al. 2008; Thalmann et al. 2009; Lagrange et al. 2009; Marois et al. 2010; Lafrenière, Javawardhana \& van Kerkwijk 2010). On the other hand, it has been suggested that disk fragmentation can also explain the formation of brown dwarfs (Stamatellos \& Whitworth 2009a; Stamatellos et al. 2011) or multiple stellar systems (Machida et al. 2008; Kratter et al. 2010). 
The ultimate fate of the clumps depends upon their orbital and internal evolution. If the migration timescale is very small, the clump accretes onto the central protostar and eventually disappears. On the other hand, if the collapse timescale of the clump is sufficiently long, dust sedimentation may cause planetary embryos to form inside the clump (Navakshin 2010a).

Although the orbital and internal evolution of clumps in circumstellar disk is clearly important, a limited number of studies exist on the topic. Baruteau, Meru \& Paardekooper (2011) investigated the orbital evolution of massive planets formed by disk fragmentation. They showed that the planets rapidly migrate inward on a type I migration timescale (Tanaka, Takeuchi \& Ward 2002). Adopting an analytical approach, Nayakshin (2010b) showed that the collapse timescale of the clumps is $\gtrsim 10^{4}$ years, considerably longer than the orbital timescale at $r \sim 100 \mathrm{AU}$ (approximately one thousand years). However, Nayakshin used a simplified opacity model, and he ignored further mass accretion onto the clumps from the disk. Recently, Galvagni et al. (2012) investigated the internal evolution of clumps which were extracted from three-dimensional (3D) global disc simulations. They reported a collapse timescale of about several thousand years, shorter than the estimates of Navakshin (2010b). However, these authors similarly neglected further mass accretion onto the clumps.

To investigate the internal evolution of clumps permitting realistic gas accretion from the disk, we must simultaneously calculate the evolution of both disk and clumps. Furthermore, appropriate treatment of radiative transfer and a realistic equation of state are crucial in studies of both the disk fragmentation (Bolev et al. 2007) and internal evolution of the clumps. Since clump evolution cannot be modeled assuming the thin disk approximation, 3D simulations are required. Two-dimensional simulations of the circumstellar disk would also overestimate the extent of disk fragmentation (T. Tsuribe, private communication). Stamatellos \& Whitworth (2009b), who investigated the internal evolution of clumps in 3D radiative $\mathrm{SPH}$ simulations, reported that clumps collapse on a timescale of $10^{3}-10^{4}$ years. However, they initially assumed a massive isolated disk and it is unclear whether such a disk can be realized during the star formation process.

To realize a self-consistent study of disk fragmentation and clump evolution, we conducted a 3D radiation hydrodynamics simulation initiated from gravitational collapse of a molecular cloud core. Using this approach, we can follow the formation of a central protostar, disk and its fragmentation. We can also follow the orbital and internal evolution of clumps with a realistic mass accretion from the disk onto them.

In this study, we ignore the magnetic field and focus on the effects of radiative cooling on the evolution of circumstellar disk and the internal structure of clumps. Note however, that magnetic field may play an important role in the formation and evolution of the circumstellar disk, because it can efficiently transfer angular momentum (Mellon \& Li 2008; Hennebelle \& Ciardi 2009; $\quad$ Inutsuka, Machida \& Matsumoto 2010; Li, Krasnopolsky \& Shang

2011; Machida, Inutsuka \& Matsumoto 2011; Joos. Hennebelle \& Ciardi 2012; Seifried et al. 2013). We

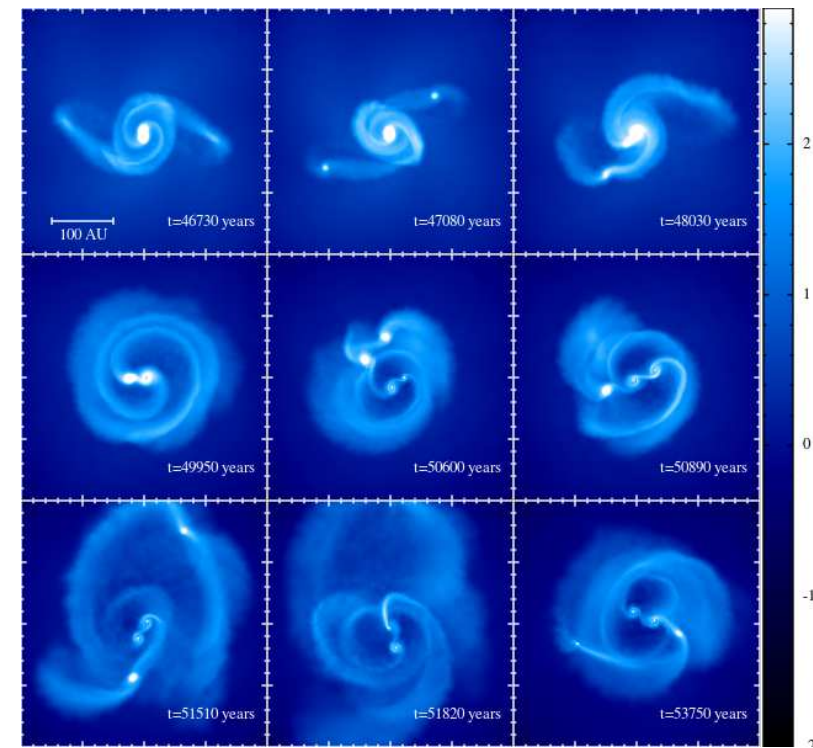

Figure 1. Time sequence of the surface density at the cloud center, viewed face-on. The elapsed time after the cloud core begins to collapse is shown in each panel.

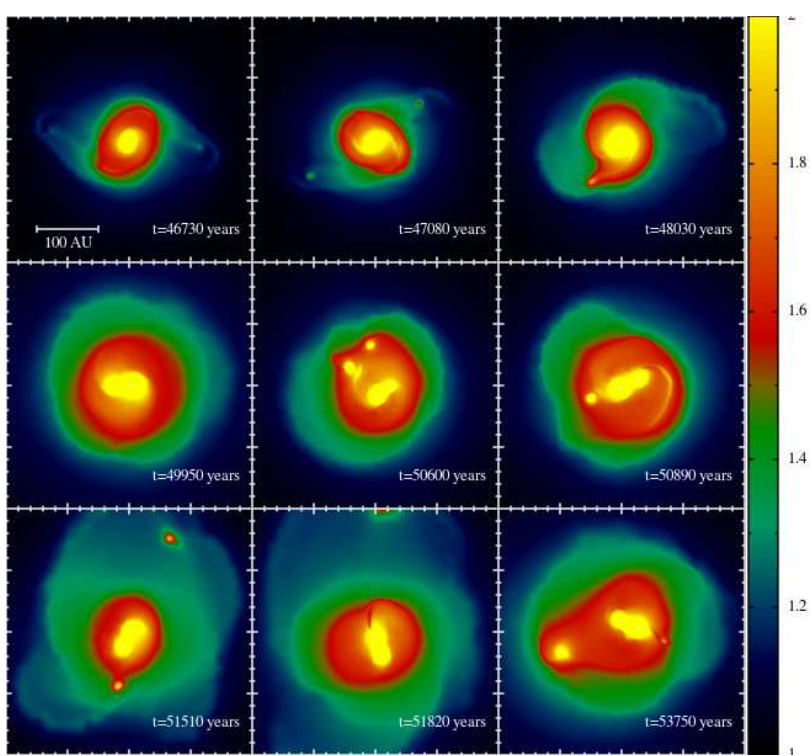

Figure 2. Same as in Fig 1 but the gas temperature is plotted.

will describe the effect of magnetic field in our subsequent paper using new numerical methods for smoothed particle magnetohydrodynamics (SPMHD) (Iwasaki \& Inutsuka 2011; Tsukamoto, Iwasaki \& Inutsuka 2013).

This paper is organized as follows. In $\S 2$, we summarize the protostar formation process in a molecular cloud core and introduce the relevant terminology. The numerical method and initial condition are described in $\S 3$, while $\S 4$, presents the results. The paper concludes with a discussion in $\S 5$. 


\section{FROM CLOUD CORE TO PROTOSTAR}

For later convenience, we summarize protostar formation process in the spherically symmetric cloud core and introduce the relevant terminology. The property of clumps formed in the disk are similar to the first core which is hydrostatic core formed during star formation. Thus, the internal evolution of the clump can be suitably described using the terminology of star formation (for more complete descriptions of the protostar formation process, see Larson 1969; Masunaga \& Inutsuka 2000; Inutsuka 2012, and references therein).

When the gravitational energy dominates the thermal energy around the center of the core, the cloud core begins to contract and the central density increases. While the radiative cooling due to dust thermal emissions overwhelms the compressional heating, the gas evolves isothermally, maintaining the temperature at about $10 \mathrm{~K}$ (isothermal collapse phase). The inner dense region collapses faster than the envelope because the timescale of gravitational collapse is a decreasing function of density, causing runaway gravitational collapse.

At sufficiently high density $\left(\rho_{\text {ad }} \sim 10^{-13} \mathrm{~g} \mathrm{~cm}^{-3}\right)$, the compressional heating catches up with radiative cooling and the isothermal collapse terminates. The gas evolves almost adiabatically within the density range $10^{-13} \mathrm{~g} \mathrm{~cm}^{-3} \lesssim \rho \lesssim$ $10^{-8} \mathrm{~g} \mathrm{~cm}^{-3}$. The gas temperature rises under adiabatic contraction with the adiabatic index, $\gamma=5 / 3$ for $T \lesssim 100 \mathrm{~K}$ and with $\gamma=7 / 5$ for $T \gtrsim 100 \mathrm{~K}$. During this phase, thermal energy dominates over gravitational energy and a quasihydrostatic core forms. We refer to this quasi-hydrostatic core as the first core.

Once the central temperature of the first core reaches $\sim 1500 \mathrm{~K}$, the hydrogen molecules begin to dissociate. This endothermic reaction promotes a second round of gravitational collapse at $10^{-8} \mathrm{~g} \mathrm{~cm}^{-3}<\rho<10^{-3} \mathrm{~g} \mathrm{~cm}^{-3}$, known as the second collapse. Finally, when the molecular hydrogen is completely dissociated, the gas again evolves adiabatically with $\gamma=5 / 3$ to form another hydrostatic core called as the second core. The initial mass of and radius of the second core are $M \sim 10^{-3} M_{\odot}$ and $r \sim 1 R_{\odot}$, respectively. Only a small proportion of the first core collapses into the second core. Therefore, the mass of the second core is rapidly increased by mass accretion from the remnant of the first core.

\section{NUMERICAL METHOD AND INITIAL CONDITION}

In this study, we extend the simulation code used in our previous studies (Tsukamoto \& Machida 2011, 2013), to include radiative transfer with flux-limited diffusion approximation according to Whitehouse \& Bate (2004); Whitehouse, Bate \& Monaghan (2005). Unlike these works, we adopted standard explicit scheme for the gas pressure and the artificial viscosity in the gas energy equation. We adopted the equation of state (EOS) used in Tomida et al. (2013), which involves seven species: $\mathrm{H}_{2}, \mathrm{H}, \mathrm{H}^{+}, \mathrm{He}, \mathrm{He}^{+}, \mathrm{He}^{++}, \mathrm{e}^{-}$. Molecular hydrogen is assumed as a 3:1 mixture of ortho- and para- hydrogen and the translational, rotational and vibrational degrees of freedom are taken into account. The hydrogen and helium mass fractions are $X=0.7$ and $Y=0.28$, respectively.
We used dust opacity table provided by Semenov et al. (2003) and gas opacity table by Ferguson et al. (2005). We did not use individual time-step technique for this work and all particles were updated simultaneously. When the density exceeds the threshold density, $\rho_{\mathrm{thr}}\left(5 \times 10^{-8} \mathrm{~g} \mathrm{~cm}^{-3}\right)$, a sink particle was introduced. Around $\rho_{\mathrm{thr}}$, the gas temperature reaches the dissociation temperature of molecular hydrogen $(T \sim 1500 \mathrm{~K})$ and the second collapse begins in the clump. Therefore, we can follow the thermal evolution of the clump just prior to second collapse. The sink radius was set as $r_{\text {sink }}=2 \mathrm{AU}$.

The initial condition is an isothermal cloud core of uniform density, rigidly rotating with angular velocity $\Omega_{0}=$ $1.4 \times 10^{-13} \mathrm{~s}^{-1}$. The mass, radius and temperature of the core are $M=1 M_{\odot}, r=3933 \mathrm{AU}$ and $T=10 \mathrm{~K}$, respectively. The resultant density is $\rho_{0}=2.3 \times 10^{-18} \mathrm{~g} \mathrm{~cm}^{-3}$. The initial condition was subjected to a density perturbation, $\delta \rho(=0.01 \times \cos 2 \phi)$. The ratios of thermal to gravitational energy $\alpha_{0}\left(\equiv E_{\mathrm{t}} / E_{\mathrm{g}}\right)$ and rotational to gravitational energy $\beta_{0}\left(\equiv E_{\mathrm{r}} / E_{\mathrm{g}}\right)$ are $\alpha_{0}=0.384$ and $\beta_{0}=0.01$, respectively, where $E_{\mathrm{t}}, E_{\mathrm{r}}$ and $E_{\mathrm{g}}$ denote the thermal, rotational and gravitational energy of the initial cloud core. These values are consistent with the results of recent $3 \mathrm{D}$ MHD simulation of molecular cloud and involved core formation (Inoue \& Inutsuka 2012). The cloud core was modeled with about $530000 \mathrm{SPH}$ particles.

\section{RESULTS}

\subsection{Overview of evolution}

Starting from a prestellar core, we calculated the disk and clump evolutions to $\sim 6000$ years following the first fragmentation. Figure 1 shows the surface density evolution at the center of the cloud core. This figure indicates that many clumps can form in the circumstellar disk during the early stages of disk evolution. Because the clumps connect smoothly to the disk, they are not always clearly delineated. In this paper, we defined a clump as a gaseous object whose central density, $\rho_{c}$ is $10^{-11} \mathrm{~g} \mathrm{~cm}^{-3}<\rho_{c}<\rho_{\mathrm{thr}}=$ $5 \times 10^{-8} \mathrm{~g} \mathrm{~cm}^{-3}$.

When the central density exceeds $10^{-11} \mathrm{~g} \mathrm{~cm}^{-3}$, the clumps are clearly distinguished from the background gas (of typical density, $\rho \lesssim 10^{-12} \mathrm{~g} \mathrm{~cm}^{-3}$ ). At $\rho_{c} \sim \rho_{\mathrm{thr}}$, the central temperature exceeds the dissociation temperature of the hydrogen molecule $(T \sim 1500 \mathrm{~K})$ and the second collapse begins. We also defined an epoch of clump formation as the time when its central density exceeds $10^{-11} \mathrm{~g} \mathrm{~cm}^{-3}$, although gravitational contraction begins at lower density. Since the central first core is not formed in the disk, its thermal evolution is different from that of the other clumps. Therefore, we do not regard it as a clump. The central density of the first core exceeded $\rho_{\text {thr }}$ at $t=47500$ years (between Fig. 1 $b$ and Fig. 1 $c$ ) and the first sink particle was introduced at this stage.

Two clumps formed (Fig. 1b) by the disk fragmentation. They rapidly migrated to the center and accreted onto the sink particle (Fig. 1 $c$ ). During this migration, a new clump formed at $r \sim 80-100 \mathrm{AU}$ (Fig. 1 $c$ ) and similarly migrated rapidly to the center. As it journeyed, the new clump gathered mass from the disk, increasing its central density and 


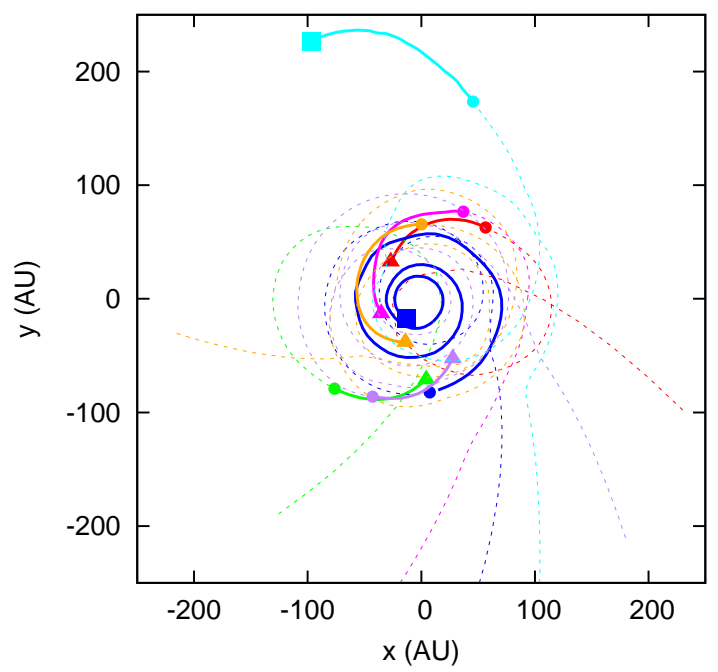

Figure 3. The orbits of the representative gas particles are shown. Dashed and solid lines represent the orbits before and after the clump formation $\left(\rho_{c}>10^{-11} \mathrm{~g} \mathrm{~cm}^{-3}\right)$, respectively. Symbols mark the positions where the clumps forms (circles), where the clump central density begins to decrease (triangles), and where the second collapse begins in the clump (or the sink particles are inserted; square).

temperature (Fig. 1 d). The central density of the clump exceeded $\rho_{\text {thr }}$ and the second sink particle was introduced between Fig. 1 $d$ and $e$. The two sink particles were gravitationally bound and formed a binary. As seen in Fig. 11e- $g$, clump formation continued in the circumbinary disk throughout the simulation time, despite the presence of the second sink (Fig. 1 $k-g$ ). Most of the clumps rapidly accreted onto the sink particles and disappeared. However, one clump formed relatively far from both sink particles (see upper region of Fig. 19) survived. The central density of this clump exceeded $\rho_{\text {thr }}$ and a third sink particle was introduced.

Seven clumps formed within 6000 years after the first clump formation, of which five migrated to the central region and finally accreted onto the sink particles. The two clumps survived to second collapse. At the end of the calculation, the masses of the sink particles (in order of decreasing age) were $0.2 M_{\odot}, 0.15 M_{\odot}$, and $0.06 M_{\odot}$, respectively.

Figure 2 shows the gas temperature at the epochs indicated in Fig. 1. Collectively, Figs. 1 and 2 reveal a weak correlation between density and temperature. The gas temperature is rather determined by the gravitational potential. Therefore, EOSs that assume a polytropic relationship between pressure and density, $p=p(\rho)$ (or the barotropic approximation) do not appropriately describe the thermal evolution of the disk. We also observe that clumps form in the outer cold regions of the disk, where the gas temperature is several tens Kelvin. Once a clump has formed, its temperature rapidly increases to higher than $100 \mathrm{~K}$ under compressional heating.

\subsection{Orbital and internal evolution of clumps}

In this subsection, we investigate the orbital and thermal evolution of the clumps. We also investigate the orbital and thermal history of the gas prior to clump formation by track-

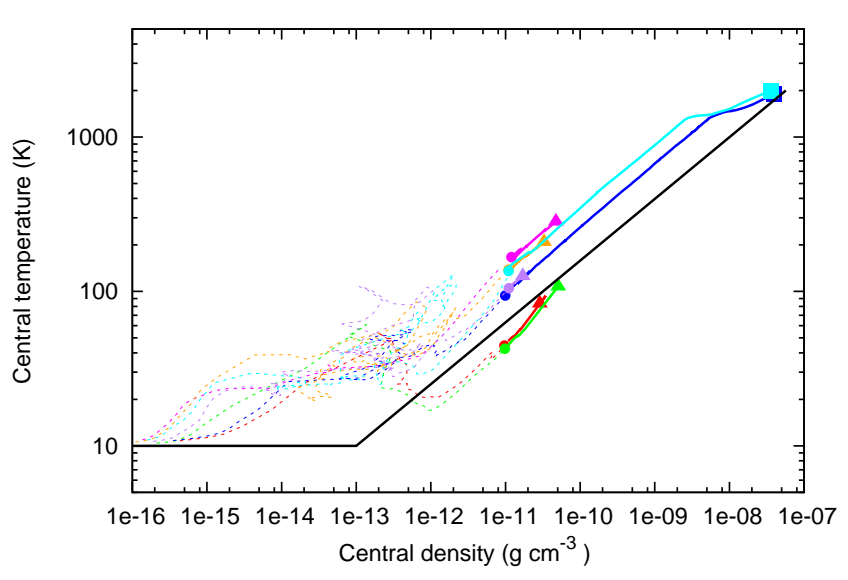

Figure 4. Temperature of representative gas particles as a function of density. Symbols are explained in the caption of Fig. 3 The clump that is identical in Figs. 3 and 4 is indicated by the same color. The black solid line plots the typical evolution of the barotropic approximation.

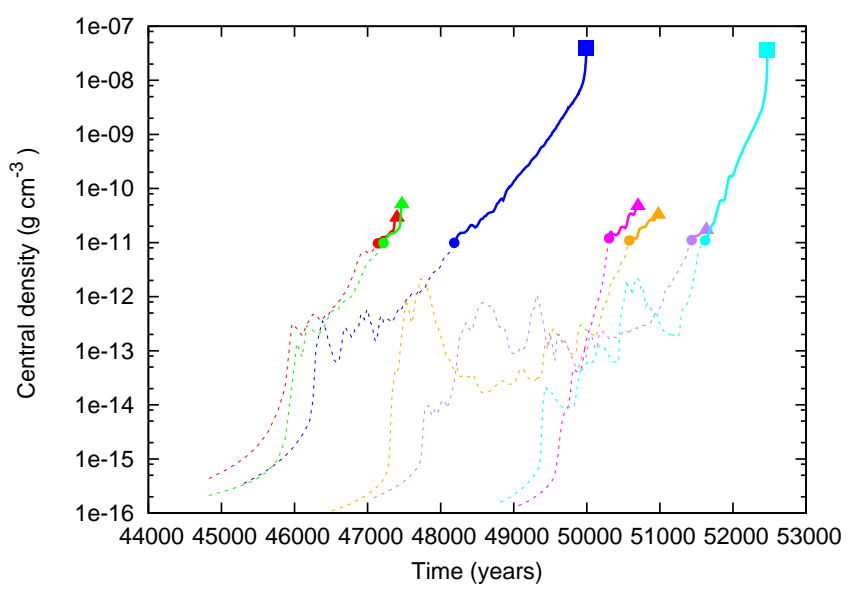

Figure 5. Density of representative gas particles as a function of elapsed time. Symbols are explained in the caption of Fig. 3 The clump that is identical in Figs. 3 and 5 is indicated by the same color.

ing the fluid element at the center of each clump. To this end, we identify representative gas particles that reside at the center of each clump. When a clump undergoes a second collapse, the representative gas particle is defined as the gas particle which has maximum density immediately before the sink particle is introduced. If a clump is tidally disrupted and accreted onto the sink particle, its central density begins to decrease at some epoch. In this case, the representative gas particle is defined as the particle which has the maximum density in the clump, at the time when the central density of clump is maximum. With this definition, the representative gas particle traces the evolution of the center of each clump.

Figure 3 shows the orbits of the representative gas particles. In this figure, the orbits of formed clumps (particle densities exceeding $10^{-11} \mathrm{~g} \mathrm{~cm}^{-3}$ ), are plotted with solid lines, while the preceding orbits are plotted with dashed lines. According to Fig. 3. the gas particles orbit within the disk for several orbital periods after they have accreted onto the disk. 
The ensuing gravitational contraction leads to clump formation. Most of the clumps are destroyed by tidal disruption and accrete onto the sink particle(s) in less than one orbital period. A notable exception is the clump shown by the cyan line. The gas was kicked out by the gravitational interaction between the binary and the clump formed relatively far from the binary. This clump was granted sufficient time for second collapse.

Figure 4 shows the temperature evolution of the representative gas particles as a function of density. In this figure, a typical temperature evolution of the barotropic approximation is shown for comparison (see, for example, Bate 2011).

Unlike the barotropic approximation, the gas temperature increases to several tens Kelvin at $\rho=10^{-15}-$ $10^{-14} \mathrm{~g} \mathrm{~cm}^{-3}$. While the gas particles orbit in the disk, they undergo the complex density and temperature evolution around $\rho \sim 10^{-14}-10^{-12} \mathrm{~g} \mathrm{~cm}^{-3}$. The temperature of the gas can increase to $100 \mathrm{~K}$ due to the heating caused by the gravitational instability. Once the density exceeds $\gtrsim 10^{-12} \mathrm{~g} \mathrm{~cm}^{-3}$, the gas becomes adiabatic and further evolution of the central temperature of the clumps consistent with adiabatic contraction. However, the complex thermal evolution at densities around $\rho \sim 10^{-14}-10^{-12} \mathrm{~g} \mathrm{~cm}^{-3}$ induces the large variance of the central entropies. As described above, most of the clumps are tidally disrupted prior to the second collapse. However, in two instances (clump evolution shown by blue and cyan lines in Figs. 3 and 4), the central temperature reaches the dissociation temperature of the molecular hydrogen, and second collapse is initiated. Dissociation is revealed by shallower slope at $T \gtrsim 1500$ $\mathrm{K}$ in Fig. 4

The collapse timescales of the clumps (timescales required for second collapse) are of particular interest because some interesting planet formation scenarios assume the long collapse timescales of the clumps (e.g., Nayakshin 2010a; Cha \& Navakshin 2011). Long collapse timescales also enable direct observation of the clumps. Figure 5 shows how the density of the representative gas particles evolves over time. When the gas accretes onto the disk, its density rapidly increases from $\rho \sim 10^{-16}$ to $10^{-13} \mathrm{~g} \mathrm{~cm}^{-3}$ and oscillates between $\rho \sim 10^{-14}-10^{-12} \mathrm{~g} \mathrm{~cm}^{-3}$ within the disk. Gravitational contraction (occurring at $\rho \gtrsim 10^{-12} \mathrm{~g} \mathrm{~cm}^{-3}$ ) is accompanied by a rapid increase in density. Although most of the clumps disappeared within several hundred years, two clumps survived and collapsed over a timescale of $1000-$ 2000 years (blue and cyan lines). This timescale is much shorter than that estimated by Nayakshin (2010b).

\subsection{Structure of clumps}

In figure 6] for the two clumps that survived to second collapse, temperature is plotted as a function of density at different epochs (the clumps evolve from the circles to the rhombi in the figure) to investigate how the clump structure evolves. The profiles within $10 \mathrm{AU}$ from the center of the clumps are shown. The clump that collapses at $t=50000$ years (blue lines in figure 3. 4. 5) is referred as clump 1, while that collapsing at $t=52500$ years (cyan lines in figure 3, 4, 5) is denoted clump 2. The polytropic relationship,
$T \propto \rho^{\frac{1}{n}}(n=3,4,5)$, where $\rho$ and $T$ are the density and temperature, respectively, is plotted for comparison.

The clump structures are adequately modeled by the polytrope of index $n \sim 3$ at central temperatures, $T_{c}$ is $\lesssim 100$ $\mathrm{K}$. As the central temperature increases, the profiles become shallower, and they can be represented with the polytropes of $n \gtrsim 4$ at central temperatures exceeding $100 \mathrm{~K}$. However, the structure of clump 1 is distorted and a single-index polytrope yields a poor fit at $T_{c}>100 \mathrm{~K}$. On the other hand, the single-index polytrope sufficiently fits the structure of clump 2 at these temperature.

The structural difference between clumps 1 and 2 is attributable to the entropy of the accretion flow. The rapid inward migration of clump 1 (see, Fig. 3) is accompanied by rapid entropy changes of the ambient disk gas, which distort the clump structure. On the other hand, the semi-major axis of clump 2 remains relatively constant suggesting that the entropy of the accretion flow also changes little throughout the clump evolution.

As shown in Fig. 6, the entropy of the clumps increases during their evolution. This is easily understood from the increased temperature at fixed density as the clump evolves. Entropy is introduced by mass accretion from the disk. Therefore, mass accretion plays an important role in the structural evolution of the clumps. In clumps evolving solely by radiative cooling, the entropy declines over time (see, for example, Chp. 17 of Cox \& Giuli 1968).

\subsection{Mass evolution of clumps}

The mass evolution of the clumps is plotted in figure 7 The solid and dashed lines denote the masses of clumps 1 and 2, respectively. Because the clumps smoothly connect to the disk, their masses cannot be directly specified. Here, we define the mass of the clump, $M_{c}$, so that it satisfies

$3 \int_{0}^{M_{c}} \frac{p}{\rho} d M_{r}=\int_{0}^{M_{c}} \frac{G M_{r}}{r} d M_{r}$,

where $p, \rho$ and $M_{r}$ signify the pressure, density, and cumulative mass at $r$, respectively. Note that equation (1) is identical to Virial theorem, if the surface pressure is negligible and the clump is in hydrostatic equilibrium. The discretized form of equation (1) is,

$3 \sum_{j} \frac{p_{j}}{\rho_{j}} m_{j}=\sum_{j} \frac{G M_{r<r_{j}}}{r_{j}} m_{j}$,

where $p_{j}, \rho_{j}, m_{j}$ and $r_{j}$ are the pressure, density, mass, and radius from the clump center of the $j$-th particle, respectively. Summation is performed in ascending order from the particle of smallest radius. $M_{r<r_{j}}$ is the total mass within $r_{j}$. As shown in Fig. 7, the clumps continually aggregate mass throughout their evolution. Clump 1 and 2 undergo second collapse at 900 years and 1800 years after its formation, respectively. The clump mass at second collapse is $\sim 0.03 M_{\odot}$, consistent with the maximum clump mass predicted for a polytrope sphere (see, Equation (3) in $\S 5$ ).

The mass accretion rate onto the clump can be estimated from Fig. 7. The clump mass increases by approximately $0.01 M_{\odot}$ within 1000 years, yielding a mass accretion rate onto the clumps, $\dot{M}_{c} \sim 10^{-5} M_{\odot}$ years $^{-1}$. This result also accords with the theoretical estimate (see, Equation (4) in $\S 5$ ). 

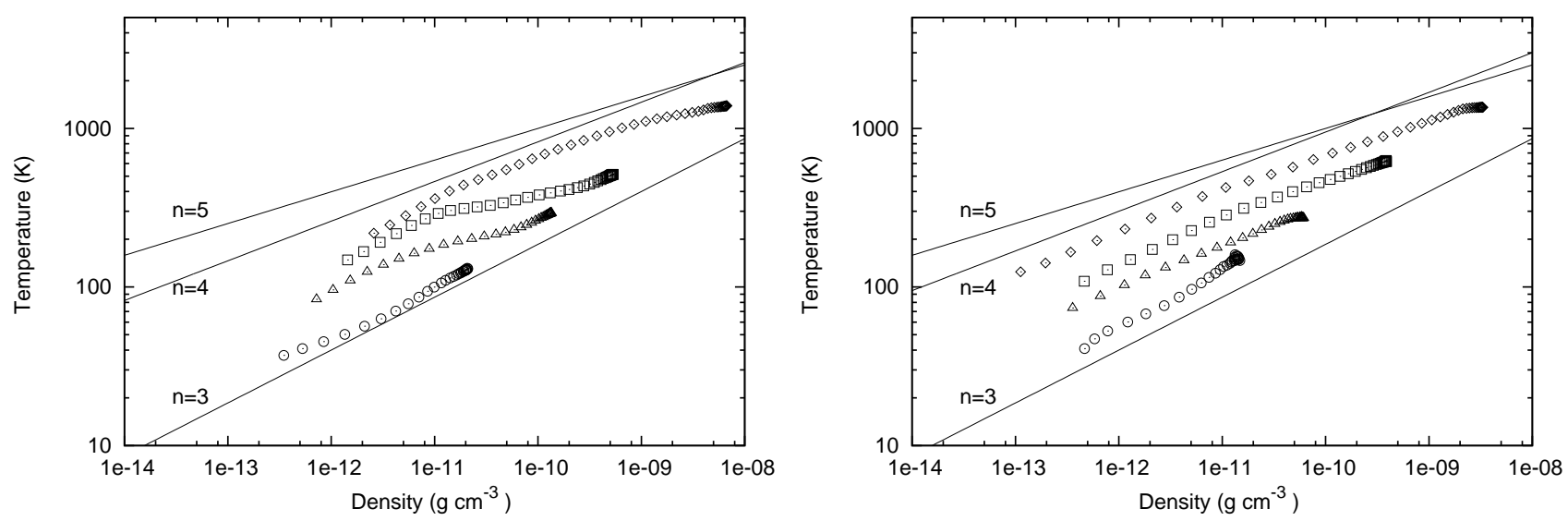

Figure 6. Profiles of clumps 1 (left) and 2 (right) in the density-temperature plane within 10 AU of the clump center. Symbols denote the profiles at different epochs. The central density of each epoch is $\rho_{c}=2.1 \times 10^{-11} \mathrm{~g} \mathrm{~cm}^{-3}$ (circles), $1.3 \times 10^{-10} \mathrm{~g} \mathrm{~cm}{ }^{-3}$ (triangles), $1.5 \times 10^{-9} \mathrm{~g} \mathrm{~cm}^{-3}$ (rectangles) and $6.7 \times 10^{-9} \mathrm{~g} \mathrm{~cm}^{-3}$ (rhombi) for clump 1 and $\rho_{c}=1.6 \times 10^{-11} \mathrm{~g} \mathrm{~cm}^{-3}(\mathrm{circles}), 6.2 \times 10^{-11} \mathrm{~g} \mathrm{~cm}^{-3}$ (triangles), $3.9 \times 10^{-10} \mathrm{~g} \mathrm{~cm}^{-3}$ (rectangles) and $3.3 \times 10^{-9} \mathrm{~g} \mathrm{~cm}^{-3}$ (rhombi) for clump 2. Solid lines show the polytropic relation, $T \propto \rho^{\frac{1}{n}}(n=3,4,5)$.

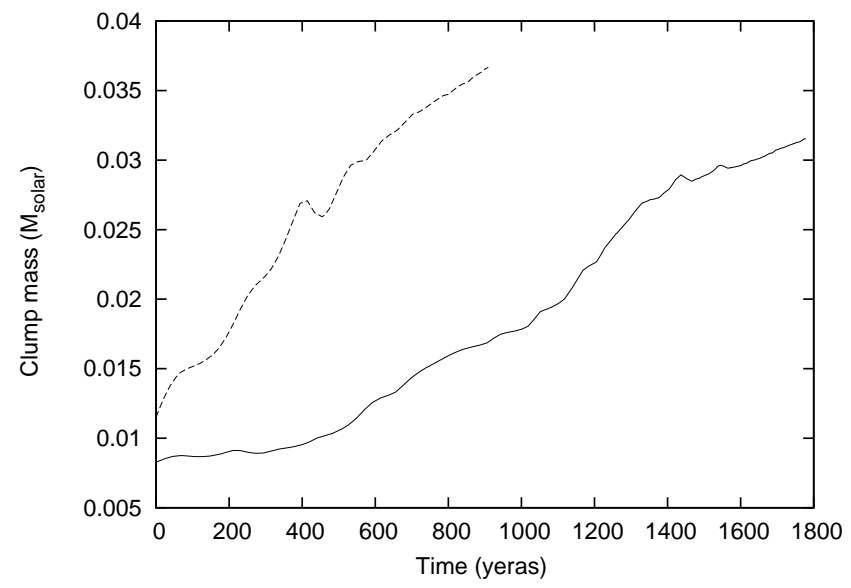

Figure 7. The mass of the clump 1 (solid) and clump 2 (dashed) as a function of the elapsed time after the clump formation.

\section{SUMMARY AND DISCUSSION}

In this paper, we performed a radiation hydrodynamics simulation using a realistic equation of state and investigated the orbital and internal evolution of clumps formed by disk fragmentation. Note that we investigated the formation and evolution of disk and clumps self-consistently.

According to our simulation, disk fragmentation and clump formation occur in the early evolution phase of the circumstellar disk. Disk fragmentation is induced by mass accretion from the infalling envelope. These results are consistent with previous works (Machida. Inutsuka \& Matsumoto 2011; Zhu et al. 2012). We show that most of the clumps rapidly migrate inward and accrete onto protostar(s). However, some clumps may survive to second collapse.

The disk gas and clump centers show the following evolutionary trends in their density and temperature. At densities of $10^{-14} \mathrm{~g} \mathrm{~cm}^{-3} \lesssim \rho \lesssim 10^{-12} \mathrm{~g} \mathrm{~cm}^{-3}$, the gas resides in the disk, where it undergoes complex thermal evolution. Heating caused by gravitational instability can raise the gas temperature to $T \sim 100 \mathrm{~K}$. When the density exceeds $\sim 10^{-12} \mathrm{~g} \mathrm{~cm}^{-3}$, gravitational contraction begins and clumps form. The central gas of the clump evolves adiabatically and further evolution typifies that of first cores (Masunaga \& Inutsuka 2000). However, reflecting the complex thermal history, the central entropy of the clumps has large deviation. Once gravitational contraction has begun, the central density increases rapidly toward second collapse within 1000 - 2000 years. We found that the clumps are adequately modeled by the polytrope spheres of index $n \sim 3$ at central temperatures $\lesssim 100 \mathrm{~K}$ and $n \gtrsim 4$ at higher central temperature. These indices are much higher than those used in Zhu et al. (2012).

Fixing the polytropic index, the clump mass can be estimated as a function of the central temperature, $T_{c}$ and central density, $\rho_{c}$. During adiabatic contraction, the entropy is constant and $T_{c} / \rho_{c}^{\gamma-1}=T_{\text {ad }} / \rho_{\text {ad }}^{\gamma-1}$, assuming constant heat capacity at constant volume $c_{v}$ and constant ratio of heat capacity $\gamma$. we define the adiabatic density, $\rho_{\text {ad }}$ and adiabatic temperature, $T_{\text {ad }}$ as the values above which the central gas in the clump evolves adiabatically. The mass of the clumps 
is then given as

$$
\begin{aligned}
M_{c}= & (n+1)^{3 / 2}\left[\frac{k_{\mathrm{B}}^{3}}{4 \pi G \mu^{3} m_{\mathrm{u}}^{3}} \frac{T_{c}^{3}}{\rho_{c}}\right]^{\frac{1}{2}}\left[-\xi^{2} \frac{d \theta}{d \xi}\right]_{\xi=\xi_{n}} \\
= & \left\{\begin{array}{r}
3.37 \times 10^{-2}(n=4) \\
2.71 \times 10^{-2}(n=3)
\end{array}\right\} \\
& \times\left(\frac{T_{c}}{1000 \mathrm{~K}}\right)^{\frac{1}{4}}\left(\frac{\rho_{\mathrm{ad}}}{10^{-14} \mathrm{~g} \mathrm{~cm}^{-3}}\right)^{-\frac{1}{2}}\left(\frac{T_{\mathrm{ad}}}{10 \mathrm{~K}}\right)^{\frac{5}{4}} M_{\odot}
\end{aligned}
$$

where, $k_{\mathrm{B}}, \mu$ and $m_{\mathrm{u}}$ are the Boltzmann constant, mean molecular weight, and atomic mass, respectively. In this estimate, $c_{v}$ and $\gamma(=7 / 5)$ are assumed constant for simplicity, and the clump mass is expressed in terms of central temperature, adiabatic density, and adiabatic temperature. Molecular hydrogen begins to dissociate at central temperatures $\sim 1000 \mathrm{~K}$, the temperature of second collapse. Thus, equation (3) indicates that second collapse occurs when the clump mass reaches $\sim 0.03 M_{\odot}$. In other words, the clump has a maximum mass and second collapse is inevitable in clumps of mass $\sim 0.03 M_{\odot}$. Therefore, the timescale of clump collapse is related to the mass accretion rate. This estimate of the maximum mass agrees favorably with our simulation results. The two clumps formed in our simulation collapsed at masses $0.036 M_{\odot}$ and $0.031 M_{\odot}$ (see Fig. 7).

We also showed that the clump entropy increases as the clump evolves. This implies that clump evolution is driven by mass accretion. By contrast, entropy decreases in clumps evolving by radiative cooling alone. The mass accretion rate onto the clump can be estimated by the BondiHoyle-Lyttleton (Bondi \& Hovle 1944) accretion rate as

$$
\begin{aligned}
\dot{M}_{c}= & \frac{2 \pi G^{2} M_{c}^{2} \rho_{\infty}}{\left(c_{\infty}^{2}+v_{\infty}^{2}\right)^{3 / 2}} \\
= & 2.7 \times 10^{-5} \\
& \times\left(\frac{M_{c}}{0.01 \mathrm{M}_{\odot}}\right)^{2}\left(\frac{\rho_{\infty}}{10^{-14} \mathrm{~g} \mathrm{~cm}^{-3}}\right)\left(\frac{\sqrt{c_{\infty}^{2}+v_{\infty}^{2}}}{400 \mathrm{~m} \mathrm{~s}^{-1}}\right)^{-3} M_{\odot} \text { year }^{-1}
\end{aligned}
$$

where, $\rho_{\infty}, c_{\infty}$, and $v_{\infty}$ are the density, sound velocity and the characteristic velocity of the gas far from the clump, respectively. Here, we assumed the typical velocity of the gas as $400 \mathrm{~m} \mathrm{~s}^{-1}$, which is close to the value of the sound velocity at $T \sim 40 \mathrm{~K}$. From the accretion rate, the timescale of second collapse is estimated as 1000 years $\left(\simeq 0.03 M_{\odot} / 2.7 \times 10^{-5} M_{\odot} \mathrm{yr}^{-1}\right)$, consistent with the collapse timescale of the simulated clumps (see, Fig. 7), but much smaller than the estimates of Nayakshin (2010b). This difference is largely attributable to the negligence of further mass accretion in the earlier study. Our simulation suggests that mass accretion is a main driver of clump collapse.

The typical luminosity of clumps in circumstellar disks has been estimated as $L \sim 10^{-3} L_{\odot}$ (see, for example, Zhu et al. 2012). Here, we emphasize that clump luminosity would significantly increase at second collapse, as the clump gas rapidly accretes onto the new born second core. The luminosity is estimated as

$$
\begin{aligned}
L_{\mathrm{acc}} & =\frac{G M_{\mathrm{pr}} \dot{M}_{\mathrm{pr}}}{R_{\mathrm{pr}}} \\
& =25 \times\left(\frac{M_{\mathrm{pr}}}{0.01 \mathrm{M}_{\odot}}\right)\left(\frac{\dot{M}_{\mathrm{pr}}}{2.4 \times 10^{-4} \mathrm{M}_{\odot} \text { year }^{-1}}\right)\left(\frac{R_{\mathrm{pr}}}{3 R_{\odot}}\right)^{-1} L_{\odot},
\end{aligned}
$$

where $M_{\mathrm{pr}}, R_{\mathrm{pr}}$, and $\dot{M}_{\mathrm{pr}}$ are the second core mass, second core radius and the mass accretion rate onto the second core, respectively. The mass accretion rate is estimated as

$$
\dot{M}_{\mathrm{pr}}=\frac{c_{s}^{3}}{G}=2.4 \times 10^{-4}\left(\frac{c_{s}}{1000 \mathrm{~m} \mathrm{~s}^{-1}}\right)^{3} \mathrm{M}_{\odot} \operatorname{year}^{-1} .
$$

$3 R_{\odot}$ is the typical radius of a young protostar (e.g., Palla \& Stahler 1993; Masunaga \& Inutsuka 2000). This luminosity increase would continue over about $M_{c} / \dot{M}_{\mathrm{pr}} \sim 100$ years.

Following second collapse, the newly formed second core accumulates further mass from the disk. The final masses of the sink particles emerging from our simulation were $0.2 M_{\odot}, 0.15 M_{\odot}$, and $0.06 M_{\odot}$ at the end of the calculation. Thus, they are rather protostars or brown dwarfs than protoplanets. This is consistent with the results of Stamatellos \& Whitworth (2009b a).

In our simulation, the formed clumps either fall onto the protostar(s) and disappear or evolve into protostars or brown dwarfs. The clump mass easily exceeds the planetary mass range $\left(\lesssim 0.01 M_{\odot}\right)$. Therefore, if disk fragmentation is responsible for the wide-orbit planets found in recent observations (e.g., Marois et al. 2010), the rapid migration to the central star should be avoided, and the mass of the clumps should be kept small. Although the latter condition is little recognized, it is problematic in explaining how distant planets can emerge from disk fragmentation. To retain small mass clump and avoid rapid inward migration, an additional mechanism that decouples the disk and the clumps may be required. We will investigate such mechanisms in a more realistic setup involving magnetic fields (see, Iwasaki \& Inutsuka 2011; Tsukamoto, Iwasaki \& Inutsuka 2013, Iwasaki in preparation)

\section{ACKNOWLEDGMENTS}

We thank T. Tsuribe, K. Iwasaki, S. Okuzumi, E.I. Vorobyov, and K. Tomida for their fruitful discussions. We also thank K. Tomida and Y. Hori to provide their EOS table to us. The snapshots were produced by SPLASH Price 2007). The computations were performed on a parallel computer, XT4 system at CfCA of NAOJ and SR16000 at YITP in Kyoto University. Y.T. is financially supported by Research Fellowships of JSPS for Young Scientists.

\section{REFERENCES}

Baruteau C., Meru F., Paardekooper S.-J., 2011, MNRAS, 416, 1971

Bate M. R., 1998, ApJ, 508, L95

-, 2011, MNRAS, 417, 2036

Boley A. C., Hartquist T. W., Durisen R. H., Michael S., 2007, ApJ, 656, L89 
Bondi H., Hoyle F., 1944, MNRAS, 104, 273

Cha S.-H., Nayakshin S., 2011, MNRAS, 415, 3319

Cox J. P., Giuli R. T., 1968, Gordon and Breach, science publishers

Ferguson J. W., Alexander D. R., Allard F., Barman T., Bodnarik J. G., Hauschildt P. H., Heffner-Wong A., Tamanai A., 2005, ApJ, 623, 585

Galvagni M., Hayfield T., Boley A., Mayer L., Roškar R., Saha P., 2012, MNRAS, 427, 1725

Gammie C. F., 2001, ApJ, 553, 174

Hennebelle P., Ciardi A., 2009, A\&A, 506, L29

Inoue T., Inutsuka S.-i., 2012, ApJ, 759, 35

Inutsuka S., 2012, Prog. Theor. Exp. Phys., 2012, 307

Inutsuka S., Machida M. N., Matsumoto T., 2010, ApJ, 718, L58

Iwasaki K., Inutsuka S., 2011, MNRAS, 418, 1668

Joos M., Hennebelle P., Ciardi A., 2012, A\&A, 543, A128

Kimura S. S., Tsuribe T., 2012, PASJ, 64, 116

Kratter K. M., Matzner C. D., Krumholz M. R., Klein R. I., 2010, ApJ, 708, 1585

Lafrenière D., Jayawardhana R., van Kerkwijk M. H., 2010, ApJ, 719, 497

Lagrange A.-M. et al., 2009, A\&A, 493, L21

Larson R. B., 1969, MNRAS, 145, 271

Laughlin G., Bodenheimer P., 1994, ApJ, 436, 335

Li Z.-Y., Krasnopolsky R., Shang H., 2011, ApJ, 738, 180

Machida M. N., Inutsuka S., Matsumoto T., 2010, ApJ, 724,1006

—, 2011, ApJ, 729, 42

Machida M. N., Tomisaka K., Matsumoto T., Inutsuka S., 2008, ApJ, 677, 327

Marois C., Macintosh B., Barman T., Zuckerman B., Song I., Patience J., Lafrenière D., Doyon R., 2008, Science, 322,1348

Marois C., Zuckerman B., Konopacky Q. M., Macintosh B., Barman T., 2010, Nature, 468, 1080

Masunaga H., Inutsuka S., 2000, ApJ, 531, 350

Mellon R. R., Li Z.-Y., 2008, ApJ, 681, 1356

Nayakshin S., 2010a, MNRAS, 408, L36

一, 2010b, MNRAS, 408, 2381

Palla F., Stahler S. W., 1993, ApJ, 418, 414

Price D. J., 2007, PASA, 24, 159

Rice W. K. M., Lodato G., Armitage P. J., 2005, MNRAS, 364, L56

Seifried D., Banerjee R., Pudritz R. E., Klessen R. S., 2013, MNRAS, 432, 3320

Semenov D., Henning T., Helling C., Ilgner M., Sedlmayr E., 2003, A\&A, 410, 611

Stamatellos D., Maury A., Whitworth A., André P., 2011, MNRAS, 413, 1787

Stamatellos D., Whitworth A. P., 2009a, MNRAS, 392, 413 —, 2009b, MNRAS, 400, 1563

Stamatellos D., Whitworth A. P., Hubber D. A., 2011, ApJ, 730, 32

Takahashi S. Z., Inutsuka S., Machida M. N., 2013, ApJ, 770,71

Tanaka H., Takeuchi T., Ward W. R., 2002, ApJ, 565, 1257

Thalmann C. et al., 2009, ApJ, 707, L123

Tomida K., Tomisaka K., Matsumoto T., Hori Y., Okuzumi S., Machida M. N., Saigo K., 2013, ApJ, 763, 6

Tsukamoto Y., Iwasaki K., Inutsuka S., 2013, MNRAS, 434, 2593
Tsukamoto Y., Machida M. N., 2011, MNRAS, 416, 591 -, 2013, MNRAS, 428, 1321

Vorobyov E. I., Basu S., 2010, ApJ, 714, L133

Vorobyov E. I., DeSouza A. L., Basu S., 2013, ApJ, 768, 131

Whitehouse S. C., Bate M. R., 2004, MNRAS, 353, 1078

Whitehouse S. C., Bate M. R., Monaghan J. J., 2005, MNRAS, 364, 1367

Zhu Z., Hartmann L., Nelson R. P., Gammie C. F., 2012, ApJ, 746, 110 